\title{
Adaptive Sliding Mode Tracking Control for Unmanned Autonomous Helicopters Based on Neural Networks
}

\author{
Min Wan (D, Mou Chen (D), and Kun Yan \\ College of Automation Engineering, Nanjing University of Aeronautics and Astronautics, Nanjing, China \\ Correspondence should be addressed to Mou Chen; chenmou@nuaa.edu.cn
}

Received 13 July 2018; Accepted 28 August 2018; Published 1 November 2018

Academic Editor: Zhile Yang

Copyright (@ 2018 Min Wan et al. This is an open access article distributed under the Creative Commons Attribution License, which permits unrestricted use, distribution, and reproduction in any medium, provided the original work is properly cited.

\begin{abstract}
In this paper, an adaptive sliding mode tracking control scheme is developed for the medium-scale unmanned autonomous helicopter with system uncertainties and external unknown disturbances. A simplified mathematical model is established, which is divided into position subsystem and attitude subsystem. The uncertainty term of the system is handled by the inherent approximation ability of the neural network. The sliding model control scheme under the backstepping frame is developed for tackling disturbances. The stability of the simplified system is proved by using the Lyapunov theory, and the tracking errors are guaranteed to be uniformly bounded. Numerical simulation results show that the proposed control strategy is effective.
\end{abstract}

\section{Introduction}

The unique flight capabilities of unmanned autonomous helicopters (UAHs), such as vertical take-off and landing, hovering, low-altitude flying, make them have an irreplaceable position in military and civil fields. The UAHs are widely used in battlefield reconnaissance, information collection, earthquake relief, and fire detection [1]. Compared with small-scale UAHs, medium-scale UAHs which weigh between $500 \mathrm{~kg}$ and $1000 \mathrm{~kg}$ have significant and remarkable advantages of high altitude, long cruise, large payload, and strong robustness [2]. However, the dynamics of the UAH are highly nonlinear, strong coupling, and underactuated. Meanwhile, autonomous flight control technology is an extremely complex system engineering, which involves flight dynamics, aerodynamics, microelectronics, image processing, wireless transmission technology, advanced control technology, and other fields. These features make the flight difficult to control. Thus, the design of highperformance flight control system is a very challenging research topic.

Recently, some efficient control methods have been explored and utilized for the UAH [3-5]. Traditional linear control methods are based on a linearized model which cannot accurately describe the actual state of the UAH. Ignoring the nonlinear term will lead to the decrease of control performance and even the instability of the system. Thus, various nonlinear control strategies have been proposed for the helicopters including feedback linearization [6], predictive control [7], backstepping control [8-11], sliding mode control (SMC) [12-16], and neural network (NN) control [17-25]. In [6], a flight controller with the approximate feedback linearization method and active disturbance rejection control was designed for the helicopter, which had good robustness. In [7], a nonlinear model predictive control combined with the disturbance observer was proposed for helicopter tracking control. Among various control methods, backstepping has become a widespread control design technique for nonlinear control systems due to a systematic design process $[8,9]$. In [10], the control design based on backstepping provided asymptotic tracking and bounded tracking for a model of the UAH. In [11], the backstepping technique combined dynamic inversion with command filters was designed for the trajectory tracking of an uncertain system. The flight environment of the UAH is always changing, so that modeling uncertainties and external 
disturbances should be taken into account during the design of flight control.

SMC has the advantages of fast response, insensitivity to system parameter changes, good adaptability to unmodeled dynamics and external disturbances, and the design is simple and easy to implement $[12,13]$. In [14], a SMC design approach was adopted to globally stabilize some underactuated systems. Literature [15] designed a sliding mode controller for the stability of the UAH in suspension and forward flight and to ensure that the system can track the expected reference trajectory. In [16], two nonlinear control methods were compared and adaptive SMC can handle sensor noise and model uncertainty better than feedback linearization control.

On the other hand, NNs have been widely used as universal approximators to compensate the unmodeled dynamics of the system [17]. In [18], an adaptive predictive control combined with a high-order $\mathrm{NN}$ observer was presented to obtain predictions of future system states, which was applied to avoid input delay and nonlinearity. In [19], an adaptive neural dynamic surface control scheme was designed for a class of time-delay systems with an unknown dead-zone input. A novel high-order NN was adopted to account for unknown nonlinearities. High-order sliding mode observer combined with NNs was applied for nonlinear purefeedback systems in [20].

In [21], an optimal output feedback controller based on the NN observer was designed for tracking the trajectory of the UAH. In [22], a nonlinear adaptive controller based on the NN was designed under a backstepping framework and tracking errors were restricted within ultimate bounds. In [23], a modified NN dynamic inversion control method for the helicopter has been presented and the inversion model was based on the linearized aerodynamics of hover. In $[24,25]$, neural control was proposed for strict-feedback systems with unknown dynamics, using the dynamic surface control. The simulation results of hypersonic flight dynamics were presented to demonstrate the effectiveness. The ability of the NN was to reconstruct and cancel the errors caused by simplifications, nonlinearity, and variations in operating conditions. Combining SMC with the $\mathrm{NN}$ can improve the control performance.

This paper is motivated by the tracking control of the UAH with the effect of unknown uncertainties and disturbances. The adaptive SMC scheme based on the NN is developed for tracking reference trajectory.

The rest of this paper is organized as follows: in Section 2, the UAH model with uncertainties and disturbances is established; in Section 3, the design processes of the adaptive SMC method with NNs are presented and stability analysis is carried out to guarantee the feasibility of the proposed controller; Section 4 provides simulations to verify the effectiveness of the proposed control method; and Section 5 concludes the paper.

\section{Problem Formulation and Preliminaries}

2.1. UAH Model. In this paper, the medium-scale UAH is regarded as a rigid-body model. Then, the simplified nonlinear dynamic model of the 6 degrees of freedom (DOF) can be expressed as follows [26]:

$$
\begin{aligned}
& \dot{P}=V, \\
& \dot{V}=\left[\begin{array}{lll}
0 & 0 & g
\end{array}\right]^{T}+\frac{1}{m} R F, \\
& \dot{\Lambda}=H \Omega, \\
& \dot{\Omega}=J^{-1}(-\Omega \times J \Omega+M),
\end{aligned}
$$

where $P=\left[x_{g}, y_{g}, z_{g}\right]^{T}$ represents the positional vector in the inertial coordinate, $V=[u, v, w]$ is velocity vector, $m$ is the mass of the helicopter, $g$ is the acceleration due to gravity, $\Omega=[p, q, r]$ is the attitude angular velocity vector, $\Lambda=[\phi, \theta, \psi]^{T}$ is the attitude angle vector in the body-fixed frame, $F$ and $M$ are external forces and moments acting on the airframe, respectively, $F \approx\left[0,0,-T_{m}\right]^{T}, T_{m}$ is the thrust generated by the main rotor, $J=\operatorname{diag}\left\{J_{x x}, J_{y y}, J_{z z}\right\}$ is the inertia matrix, $R$ is the transformation matrix from the body coordinate to the inertial coordinate, and $H$ is the attitude kinematic matrix [27].

Considering the system uncertainties and external disturbances, system (1) will be reformulated. Define $x_{11}=P$, $x_{12}=V, e_{3}=[0,0,1]^{T}, x_{1}=\left[x_{11}^{T}, x_{12}^{T}\right]^{T}$, and $u_{1}=R F$. The position dynamics of the UAH can be transformed into the following affine nonlinear form:

$$
\left\{\begin{array}{l}
\dot{x}_{11}=x_{12}, \\
\dot{x}_{12}=g e_{3}+\Delta f_{p}\left(x_{1}\right)+\frac{1}{m} u_{1}+d_{1},
\end{array}\right.
$$

where $\Delta f_{p}\left(x_{1}\right)$ indicates modeling error and $d_{1}$ indicates disturbances.

Define $x_{21}=\Lambda, x_{22}=\Omega$, and $x_{2}=\left[x_{21}^{T}, x_{22}^{T}\right]^{T}$. The attitude dynamics of the UAH can be transformed into the following affine nonlinear form:

$$
\left\{\begin{array}{l}
\dot{x}_{21}=H x_{22}, \\
\dot{x}_{22}=f_{a}\left(x_{2}\right)+\Delta f_{a}\left(x_{2}\right)+g_{a}\left(x_{2}\right) u_{2}+d_{2},
\end{array}\right.
$$

where

$$
\begin{aligned}
f_{a}\left(x_{2}\right) & =\left[\begin{array}{l}
\frac{J_{y y}-J_{z z}}{J_{x x}} q r \\
\frac{J_{z z}-J_{x x}}{J_{y y}} p r \\
\frac{J_{x x}-J_{y y}}{J_{z z}} p q
\end{array}\right], \\
g_{a}\left(x_{2}\right)=J^{-1} & \\
u_{2} & =\left[\Gamma_{\phi}, \Gamma_{\theta}, \Gamma_{\psi}\right]^{T},
\end{aligned}
$$


$u_{2}$ indicate the control input of the attitude subsystem, $\Delta f_{p}$ $\left(x_{2}\right)$ indicates modeling error, and $d_{2}$ indicates disturbances.

2.2. Preliminaries. In view of the problem of handling the UAH robust trajectory tracking control, the relevant assumptions and lemmas are described as follows.

Assumption 1 [28]. The attitude kinematic matrix $H$ is considered to be nonsingular, so that the roll angle and pitch angle always change in the range of $-(\pi / 2)<\theta<(\pi / 2)$, $-(\pi / 2)<\phi<(\pi / 2)$.

Assumption 2 [29]. The external disturbances $d_{1}$ and $d_{2}$ are unknown and bounded, and there exists positive constants $\bar{d}_{1}, \bar{d}_{2}$ such that $\left\|d_{1}\right\| \leq \bar{d}_{1}$ and $\left\|d_{2}\right\| \leq \bar{d}_{2}$.

Assumption 3 [29]. The desired trajectories are bounded, and their derivatives are bounded. Furthermore, all system states can be measured.

Lemma 1 [30]. In the case of initial bounded conditions, if there exists a $C^{1}$ continuous and positive definite Lyapunov function $V(z)$ which satisfies $\varphi_{1}(\|z\|) \leq V(z) \leq \varphi_{2}(\|z\|)$ and $\dot{V}(z) \leq-$ $\mu_{1} V(z)+\mu_{2}$, where $\varphi_{1}, \varphi_{2}: R^{n} \rightarrow R$ are class $K$ functions, $\mu_{1}$ $>0$, and $\mu_{2}>0$, then the solution is uniformly bounded.

Lemma 2 [31-33]. The NN has the inherent ability of learning and implements nonlinear mapping through training of sample data. The radical basis function NN (RBFNN) is a class of linearly parameterized NNs, which is widely employed to approximate the continuous unknown function $f(Z): R^{q} \rightarrow$ $R$. It can be represented in the form of

$$
f(Z)=\widehat{\Theta}^{T} \Phi(Z)+\xi(Z)
$$

where $Z=\left[z_{1}, z_{2}, \ldots, z_{q}\right]^{T} \in R^{q}$ is the input vector of the $N N$, $\widehat{\Theta} \in R^{p}$ is a weight vector of the NN, $\xi$ is the approximation error which satisfies $|\xi(Z)| \leq \bar{\xi}, \bar{\xi}$ is an unknown bound parameter, and $\Phi(Z)=\left[\phi_{1}(Z), \phi_{2}(Z), \ldots, \phi_{p}(Z)\right]^{T} \in R^{p}$ is the Gaussian radial basis function which can be chosen as the form

$$
\phi_{i}(Z)=\exp \left[-\left(Z-\rho_{i}\right)^{T} \frac{\left(Z-\rho_{i}\right)}{\varsigma_{i}^{2}}\right], \quad i=1,2, \ldots, p
$$

where $\rho_{i}$ is the center and $\varsigma_{i}$ is the width of the hidden layer neuron.

The continuous function $f(Z)$ can be smoothly approximated over the compact set $\Omega_{Z}$ to arbitrary precision by RBFNN as

$$
f(Z)=\Theta^{* T} \Phi(Z)+\xi^{*}(Z), \quad \forall Z \in \Omega_{Z} \subset R^{q},
$$

where $\xi^{*}(Z)$ is the least approximation error in the special case of $\widehat{\Theta}=\Theta^{*}$ and the optimal weight value $\Theta^{*}$ can be defined as

$$
\Theta^{*}=\arg \min _{\widehat{\Theta} \in S_{f}}\left[\sup _{Z \in \Omega_{Z}}|\widehat{f}(Z \mid \widehat{\Theta})-f(Z)|\right] \text {, }
$$

where $S_{f}=\{\widehat{\Theta}:\|\widehat{\Theta}\| \leq \bar{\Theta}\}$ is a valid field of the estimate parameter $\widehat{\Theta}$ and $\bar{\Theta}$ is a design constant.

\section{Adaptive Sliding Mode Tracking Controller Design}

Borrowing the backstepping control method, the innerouter-loop control scheme is adopted in this section. The position loop is considered as an outer loop; then, the attitude loop is considered as an inner loop.

3.1. Scheme of Position Control. In this section, an adaptive SMC based on RBFNN will be established for the UAH position system.

Step 1. Consider (2) and define the position and velocity tracking errors

$$
\begin{aligned}
& z_{11}=x_{11}-x_{1 d}, \\
& z_{12}=x_{12}-\alpha_{1},
\end{aligned}
$$

where $x_{1 d}$ is the desired position vector and $\alpha_{1}$ as the virtual control law will be defined in the next step.

Differentiating $z_{11}$, we obtain

$$
\dot{z}_{11}=\dot{x}_{11}-\dot{x}_{1 d}=x_{12}-\dot{x}_{1 d} .
$$

Consider the Lyapunov function candidate

$$
V_{11}=\frac{1}{2} z_{11}^{2}
$$

Its time derivative is

$$
\dot{V}_{11}=z_{11}^{T} \dot{z}_{11}=z_{11}^{T}\left(x_{12}-\dot{x}_{1 d}\right)
$$

Choose the following virtual control law as

$$
\alpha_{1}=-K_{1} z_{11}+\dot{x}_{1 d}
$$

where $K_{1}=K_{1}^{T}>0$ is a design parameter. Substituting (10) and (14) into (11), we have

$$
\dot{z}_{11}=z_{12}+\alpha_{1}-\dot{x}_{1 d}=-K_{1} z_{11}+z_{12} .
$$

Step 2. Consider the second equation in (2). $\Delta f_{p}\left(x_{1}\right)$ is unknown function, so that the RBFNN is adopted to approximate $\Delta f_{p}\left(x_{1}\right)$. Thus, we obtain

$$
\Delta f_{p}\left(x_{1}\right)=\Theta_{1}^{* T} \Phi\left(x_{1}\right)+\xi_{1}
$$

where $\Theta_{1}^{*}$ is the optimal weight value and $\xi_{1}$ is the least 
approximation error of RBFNN, which satisfies $\left\|\xi_{1}\right\| \leq \bar{\xi}_{1}$ with $\bar{\xi}_{1}>0$. Define $\varepsilon_{1}=\xi_{1}+d_{1}$. The external disturbance $d_{1}$ is bounded, which satisfies $\left\|d_{1}\right\| \leq \bar{d}_{1}$ with $\bar{d}_{1}>0$. Therefore, we have that $\left\|\varepsilon_{1}\right\| \leq \beta_{1}, \beta_{1}$ is a positive constant.

Substituting (16) into the second equation in (2) results in

$$
\dot{x}_{12}=g e_{3}+\Theta_{1}^{* T} \Phi\left(x_{1}\right)+\frac{1}{m} u_{1}+\varepsilon_{1} .
$$

Differentiating $z_{12}$ and invoking (20) yield

$$
\dot{z}_{12}=\dot{x}_{12}-\dot{\alpha}_{1}=g e_{3}+\Theta_{1}^{* T} \Phi\left(x_{1}\right)+\frac{1}{\mathrm{~m}} u_{1}+\varepsilon_{1}-\dot{\alpha}_{1}
$$

Define the sliding surface as

$$
s_{1}=c_{1} z_{11}+z_{12}
$$

where $c_{1}=\operatorname{diag}\left\{c_{11}, c_{12}, c_{13}\right\}>0$ is a design parameter.

Then, the time derivative of $s_{1}$ is

$$
\dot{s}_{1}=c_{1} \dot{z}_{11}+\dot{z}_{12}
$$

Choose the Lyapunov function candidate

$$
V_{12}=\frac{1}{2} s_{1}^{2}
$$

Its time derivative is

$$
\begin{aligned}
V_{12} & =s_{1}^{T} \dot{s}_{1}=s_{1}^{T}\left(c_{1} \dot{z}_{11}+\dot{z}_{12}\right) \\
& =s_{1}^{T}\left(c_{1} \dot{z}_{11}+g e_{3}+\Theta_{1}^{* T} \Phi\left(x_{1}\right)+\frac{1}{m} u_{1}+\varepsilon_{1}-\dot{\alpha}_{1}\right) .
\end{aligned}
$$

Choose the Lyapunov function candidate

$$
V_{1}=\frac{1}{2} z_{11}^{2}+\frac{1}{2} s_{1}^{2}+\operatorname{tr}\left(\tilde{\Theta}_{1}^{T} \Gamma_{1}^{-1} \tilde{\Theta}_{1}\right)
$$

where $\Gamma_{1}=\Gamma_{1}^{T}>0$ is a design parameter, $\widehat{\Theta}_{1}$ is the estimate value of $\Theta_{1}^{*}$, and $\tilde{\Theta}_{1}=\Theta_{1}^{*}-\widehat{\Theta}_{1}$ is the estimate error. The time derivative of $V_{1}$ is

$$
\begin{aligned}
\dot{V}_{1} & =z_{11}^{T} \dot{z}_{11}+s_{1}^{T} \dot{s}_{1}+\operatorname{tr}\left(\tilde{\Theta}_{1}^{T} \Gamma_{1}^{-1} \dot{\tilde{\Theta}}_{1}\right) \\
& =-z_{11}^{T} K_{1} z_{11}+z_{11}^{T} z_{12}+s_{1}^{T} \dot{s}_{1}+\operatorname{tr}\left(\tilde{\Theta}_{1}^{T} \Gamma_{1}^{-1} \dot{\tilde{\Theta}}_{1}\right) .
\end{aligned}
$$

Choose the following SMC law as

$$
\begin{gathered}
u_{1}=m\left[c_{1}\left(K_{1} z_{11}-z_{12}\right)-g e_{3}-\widehat{\Theta}_{1}^{T} \Phi\left(x_{1}\right)\right. \\
\left.+\dot{\alpha}_{1}-z_{11}-h_{1} s_{1}-\eta_{1} \operatorname{sgn}\left(s_{1}\right)\right],
\end{gathered}
$$

where $h_{1}$ and $\eta_{1}$ are positive constants and $\eta_{1} \geq \beta_{1}$.
Substituting (25) into (24), we obtain

$$
\begin{aligned}
\dot{V}_{1}= & -z_{11}^{T} K_{1} z_{11}+z_{11}^{T} z_{12}+s_{1}^{T}\left(-z_{11}-h_{1} s_{1}-\eta_{1} \operatorname{sgn}\left(s_{1}\right)\right. \\
& \left.+\varepsilon_{1}+\tilde{\Theta}_{1}^{T} \Phi\left(x_{1}\right)\right)+\operatorname{tr}\left(\tilde{\Theta}_{1}^{T} \Gamma_{1}^{-1} \dot{\tilde{\Theta}}_{1}\right) \\
= & -z_{11}^{T}\left(K_{1}+c_{1}\right) z_{11}+s_{1}^{T}\left(-h_{1} s_{1}-\eta_{1} \operatorname{sgn}\left(s_{1}\right)+\varepsilon_{1}\right) \\
& +s_{1}^{T} \tilde{\Theta}_{1}^{T} \Phi\left(x_{1}\right)+\operatorname{tr}\left(\tilde{\Theta}_{1}^{T} \Gamma_{1}^{-1} \dot{\tilde{\Theta}}_{1}\right) .
\end{aligned}
$$

The time derivative of $\tilde{\Theta}_{1}$ is

$$
\dot{\tilde{\Theta}}_{1}=\dot{\Theta}_{1}^{*}-\dot{\widehat{\Theta}}_{1}=-\dot{\widehat{\Theta}}_{1} .
$$

Then, we obtain

$$
\operatorname{tr}\left(\tilde{\Theta}_{1}^{T} \Gamma_{1}^{-1} \dot{\tilde{\Theta}}_{1}\right)=-\operatorname{tr}\left(\tilde{\Theta}_{1}^{T} \Gamma_{1}^{-1} \dot{\widehat{\Theta}}_{1}\right) .
$$

The updated law of the $\mathrm{NN}$ is designed as

$$
\dot{\widehat{\Theta}}_{1}=\Gamma_{1}\left(\Phi\left(x_{1}\right) s_{1}^{T}+\gamma_{1} \widehat{\Theta}_{1}\right)
$$

where $\gamma_{1}>0$ is a design parameter.

Substituting (29) into (26), we have

$$
\begin{aligned}
\dot{V}_{1}= & -z_{11}^{T}\left(K_{1}+c_{1}\right) z_{1}+s_{1}^{T}\left(-h_{1} s_{1}-\eta_{1} \operatorname{sgn}\left(s_{1}\right)+\varepsilon_{1}\right) \\
& -\gamma_{1} \operatorname{tr}\left(\tilde{\Theta}_{1}^{T} \widehat{\Theta}_{1}\right) .
\end{aligned}
$$

Consider the following inequality:

$$
2 \operatorname{tr}\left(\tilde{\Theta}_{1}^{T} \widehat{\Theta}_{1}\right)=\left\|\tilde{\Theta}_{1}\right\|^{2}+\left\|\widehat{\Theta}_{1}\right\|^{2}-\left\|\Theta_{1}^{*}\right\|^{2} \geq\left\|\tilde{\Theta}_{1}\right\|^{2}-\left\|\Theta_{1}^{*}\right\|^{2} .
$$

Substituting (31) into (30), we have

$$
\dot{V}_{1} \leq-z_{11}^{T}\left(K_{1}+c_{1}\right) z_{11}-s_{1}^{T} h_{1} s_{1}-\frac{\gamma_{1}}{2}\left\|\tilde{\Theta}_{1}\right\|^{2}+\frac{\gamma_{1}}{2}\left\|\Theta_{1}^{*}\right\|^{2} .
$$

Remark 1 . It is well known that the control input $u_{1}$ is discontinuous due to the use of sign function $\operatorname{sgn}(\cdot)$, which may cause the chattering effect. In order to overcome this shortcoming, $\varphi(\cdot)$ is a continuous approximation of the sign function and defined as follows [34]:

$$
\varphi(s)=\frac{2}{\pi} \arctan (\mu s)
$$

where $\mu$ is a positive constant. Furthermore, as $\mu$ increases, 
the approximation error can be reduced. This approximation also allows the control law $u_{1}$ to be derivable.

Define $\delta=\operatorname{sgn}(s)-\varphi(s)$. From $\|\delta\|=\|\operatorname{sgn}(s)-\varphi(s)\| \leq 2$, we have

$$
\begin{aligned}
s_{1}^{T} & \left(-h_{1} s_{1}-\eta_{1} \varphi\left(s_{1}\right)+\varepsilon_{1}\right) \\
& =s_{1}^{T}\left(-h_{1} s_{1}-\eta_{1}(\operatorname{sgn}(s)-\delta)+\varepsilon_{1}\right) \\
& =-s_{1}^{T} h_{1} s_{1}-s_{1}^{T} \eta_{1}(\operatorname{sgn}(s)-\delta)+s_{1}^{T} \varepsilon_{1} \\
& =-s_{1}^{T} h_{1} s_{1}-s_{1}^{T} \eta_{1} \operatorname{sgn}(s)+\eta_{1} s_{1}^{T} \delta+s_{1}^{T} \varepsilon_{1} \\
& \leq-s_{1}^{T} h_{1} s_{1}+\eta_{1} s_{1}^{T} \delta \leq-s_{1}^{T} h_{1} s_{1}+\frac{1}{2} s_{1}^{T} s_{1}+\frac{1}{2} \eta_{1}^{2} \delta^{2} \\
& \leq-s_{1}^{T}\left(h_{1}-\frac{1}{2}\right) s_{1}+2 \eta_{1}^{2} .
\end{aligned}
$$

The inequality (32) can be written as

$$
\dot{V}_{1} \leq-\hbar_{1} V_{1}+\ell_{1}
$$

where $\hbar_{1}=\min \left\{\lambda_{\min }\left(K_{1}+c_{1}\right), h_{1}-(1 / 2), \gamma_{1} / 2\right\}$ and $\ell_{1}=$ $\left(\gamma_{1} / 2\right)\left\|\Theta_{1}^{*}\right\|^{2}+2 \eta_{1}^{2}$.

3.2. The Calculation of the Reference Attitude Angle. Considering the underactuated characteristics of the UAH, it is necessary to calculate the reference roll angle and the reference pitch angle of the attitude subsystem with the virtual control input of the position subsystem.

Define $\Omega_{r}=\left[\phi_{r}, \theta_{r}, \psi_{r}\right]^{T}$ as the reference signal of the attitude subsystem, where $\psi_{r}$ is directly indicated by the signal generator given and $\phi_{r}$ and $\theta_{r}$ are obtained by the position loop output.

Considering $u_{1}=\left[u_{x}, u_{y}, u_{z}\right]^{T}$, then $\phi_{r}, \theta_{r}$, and $T_{m}$ can be expressed as follows [35]:

$$
\begin{aligned}
\theta_{r} & =\arctan \left(\frac{u_{x} \cos \psi_{r}+u_{y} \sin \psi_{r}}{u_{z}}\right), \\
\phi_{r} & =\arctan \left(\frac{\cos \theta_{r}\left(u_{x} \sin \psi_{r}-u_{y} \cos \psi_{r}\right)}{u_{z}}\right), \\
-T_{m} & =\frac{u_{z}}{\cos \phi_{r} \cos \theta_{r}} .
\end{aligned}
$$

3.3. Scheme of Attitude Control. Similar to the position system, the control law based on SMC and RBFNN will be designed to achieve the purpose of tracking the reference attitude signal.

Step 3. Consider (3) and define the tracking errors of attitude angular and angular velocity

$$
\begin{aligned}
& z_{21}=x_{21}-x_{2 d}, \\
& z_{22}=x_{22}-\alpha_{2},
\end{aligned}
$$

where $x_{2 d}$ is the desired attitude vector and $\alpha_{2}$ will be defined.
Differentiating $z_{21}$, we obtain

$$
\dot{z}_{21}=\dot{x}_{21}-\dot{x}_{2 d}=H x_{22}-\dot{x}_{2 d}
$$

Choose the Lyapunov function candidate

$$
V_{21}=\frac{1}{2} z_{21}^{2}
$$

Its time derivative is

$$
\dot{V}_{21}=z_{21}^{T} \dot{z}_{21}=z_{21}^{T}\left(H x_{22}-\dot{x}_{2 d}\right)
$$

Choose the following virtual control law

$$
\alpha_{2}=H^{-1}\left(-K_{21} z_{21}+\dot{x}_{2 d}+c_{2} H^{T} z_{21}\right) \text {, }
$$

where $K_{2}=K_{2}^{T}>0$ and $c_{2}=\operatorname{diag}\left\{c_{21}, c_{22}, c_{23}\right\}>0$ are design parameters.

Substituting (38) and (42) into (39), we have

$$
\dot{z}_{21}=H\left(z_{22}+\alpha_{2}\right)-\dot{x}_{2 d}=-K_{21} z_{21}+H z_{22}+c_{2} H^{T} z_{21} .
$$

Step 4. Similar to the position subsystem, $\Delta f_{a}\left(x_{2}\right)$ is unknown, so the RBFNN is used to approximate $\Delta f_{a}\left(x_{2}\right)$. Thus, we obtain

$$
\Delta f_{a}\left(x_{2}\right)=\Theta_{2}^{* T} \Phi\left(x_{2}\right)+\xi_{2}
$$

where $\Theta_{2}^{*}$ is the optimal weight value and $\xi_{2}$ is the least approximation error of RBFNN, which satisfies $\left\|\xi_{2}\right\| \leq \bar{\xi}_{2}$ with $\bar{\xi}_{2}>0$. Define $\varepsilon_{2}=\xi_{2}+d_{2}$. The external disturbance $d_{2}$ is bounded, which satisfies $\left\|d_{2}\right\| \leq \bar{d}_{2}$ with $\bar{d}_{2}>0$. Therefore, we have that $\left\|\varepsilon_{2}\right\| \leq \beta_{2}, \beta_{2}$ is a positive constant.

Substituting (47) into the second equation in (6) results in

$$
\dot{x}_{22}=f_{a}\left(x_{2}\right)+\Theta_{2}^{* T} \Phi\left(x_{2}\right)+g_{a}\left(x_{2}\right) u_{2}+\varepsilon_{2} .
$$

Differentiating $z_{12}$ with respect to time and invoking (48) yield

$$
\dot{z}_{22}=\dot{x}_{22}-\dot{\alpha}_{2}=f_{a}\left(x_{2}\right)+\Theta_{2}^{* T} \Phi\left(x_{2}\right)+g_{a}\left(x_{2}\right) u_{2}+\varepsilon_{2}-\dot{\alpha}_{2} .
$$

Define the sliding surface as

$$
s_{2}=c_{2} z_{21}+z_{22}
$$

where $c_{2}=\operatorname{diag}\left\{c_{21}, c_{22}, c_{23}\right\}>0$ is a design parameter.

Then, the time derivative of $s_{2}$ is

$$
\dot{s}_{2}=c_{2} \dot{z}_{21}+\dot{z}_{22}
$$

Choose the Lyapunov function candidate

$$
V_{22}=\frac{1}{2} s_{2}^{2}
$$


Its time derivative is

$$
\begin{aligned}
\dot{V}_{22} & =s_{2}^{T} \dot{s}_{2}=s_{2}^{T}\left(c_{2} \dot{z}_{21}+\dot{z}_{22}\right) \\
& =s_{2}^{T}\left(c_{2} \dot{z}_{21}+f_{a}\left(x_{2}\right)+\Theta_{2}^{* T} \Phi\left(x_{2}\right)+g_{a}\left(x_{2}\right) u_{2}+\varepsilon_{2}-\dot{\alpha}_{2}\right) .
\end{aligned}
$$

Consider the Lyapunov function candidate

$$
V_{2}=\frac{1}{2} z_{21}^{2}+\frac{1}{2} s_{2}^{2}+\operatorname{tr}\left(\tilde{\Theta}_{2}^{T} \Gamma_{2}^{-1} \tilde{\Theta}_{2}\right)
$$

where $\Gamma_{2}=\Gamma_{2}^{T}>0$ is a design parameter, $\widehat{\Theta}_{2}$ is the estimate value of $\Theta_{2}^{*}$, and $\tilde{\Theta}_{2}=\Theta_{2}^{*}-\widehat{\Theta}_{2}$ is the estimate error. The time derivative of $V_{2}$ is

$$
\begin{aligned}
\dot{V}_{2}= & z_{21}^{T} \dot{z}_{21}+s_{2}^{T} \dot{s}_{2}+\operatorname{tr}\left(\tilde{\Theta}_{2}^{T} \Gamma_{2}^{-1} \dot{\tilde{\Theta}}_{2}\right) \\
= & -z_{21}^{T} K_{21} z_{21}+z_{21}^{T} H z_{22}+z_{21}^{T} c_{2} H^{T} z_{21} \\
& +s_{2}^{T} \dot{s}_{2}+\operatorname{tr}\left(\tilde{\Theta}_{2}^{T} \Gamma_{2}^{-1} \dot{\tilde{\Theta}}_{2}\right) .
\end{aligned}
$$

Choose the following virtual control law as

$$
\begin{aligned}
u_{2}= & g_{a}^{-1}\left(x_{2}\right)\left[c_{2}\left(K_{21} z_{21}-H z_{22}-c_{2} H^{T} z_{21}\right)-f_{a}\left(x_{2}\right)\right. \\
& \left.-\widehat{\Theta}_{2}^{T} \Phi\left(x_{2}\right)+\dot{\alpha}_{2}-H^{T} z_{21}-h_{2} s_{2}-\eta_{2} \operatorname{sgn}\left(s_{2}\right)\right],
\end{aligned}
$$

where $h_{2}$ and $\eta_{2}$ are positive constants and $\eta_{2} \geq \beta_{2}$. Substituting (53) into (52), we obtain

$$
\begin{aligned}
\dot{V}_{2}= & -z_{21}^{T} K_{2} z_{21}+z_{21}^{T} H z_{22}+z_{21}^{T} c_{2} H^{T} z_{21}+s_{2}^{T}\left(-H^{T} z_{21}-h_{2} s_{2}\right. \\
& \left.-\eta_{2} \operatorname{sgn}\left(s_{2}\right)+\varepsilon_{2}+\tilde{\Theta}_{2}^{T} \Phi\left(x_{2}\right)\right)+\operatorname{tr}\left(\tilde{\Theta}_{2}^{T} \Gamma_{2}^{-1} \dot{\tilde{\Theta}}_{2}\right) \\
= & -z_{21}^{T} K_{21} z_{21}+z_{21}^{T} H z_{22}+z_{21}^{T} c_{2} H^{T} z_{21} \\
& -\left(c_{2} z_{21}+z_{22}\right)^{T} H^{T} z_{21}+s_{2}^{T}\left(-h_{2} s_{2}-\eta_{2} \operatorname{sgn}\left(s_{2}\right)+\varepsilon_{2}\right) \\
& +s_{2}^{T} \tilde{\Theta}_{2}^{T} \Phi\left(x_{2}\right)+\operatorname{tr}\left(\tilde{\Theta}_{2}^{T} \Gamma_{2}^{-1} \dot{\tilde{\Theta}}_{2}\right) \\
= & -z_{21}^{T} K_{21} z_{21}+s_{2}^{T}\left(-h_{2} s_{2}-\eta_{2} \operatorname{sgn}\left(s_{2}\right)+\varepsilon_{2}\right) \\
& +s_{2}^{T} \tilde{\Theta}_{2}^{T} \Phi\left(x_{2}\right)+\operatorname{tr}\left(\tilde{\Theta}_{2}^{T} \Gamma_{2}^{-1} \dot{\tilde{\Theta}}_{2}\right) .
\end{aligned}
$$

The time derivative of $\tilde{\Theta}_{2}$ is

$$
\dot{\tilde{\Theta}}_{2}=\dot{\Theta}_{2}^{*}-\dot{\widehat{\Theta}}_{2}=-\dot{\widehat{\Theta}}_{2} .
$$

The updated law of the NN is designed as

$$
\widehat{\Theta}_{2}=\Gamma_{2}\left(\Phi\left(x_{2}\right) s_{2}^{T}+\gamma_{2} \widehat{\Theta}_{2}\right)
$$

where $\gamma_{2}>0$ is a design parameter.
Substituting (56) into (54), we have

$$
\begin{aligned}
\dot{V}_{2}= & -z_{21}^{T} K_{21} z_{21}+s_{2}^{T}\left(-h_{2} s_{2}-\eta_{2} \operatorname{sgn}\left(s_{2}\right)+\varepsilon_{2}\right) \\
& -\gamma_{2} \operatorname{tr}\left(\tilde{\Theta}_{2}^{T} \widehat{\Theta}_{2}\right) .
\end{aligned}
$$

Considering the following inequality:

$$
2 \operatorname{tr}\left(\tilde{\Theta}_{2}^{T} \widehat{\Theta}_{2}\right)=\left\|\tilde{\Theta}_{2}\right\|^{2}+\left\|\widehat{\Theta}_{2}\right\|^{2}-\left\|\Theta_{2}^{*}\right\|^{2} \geq\left\|\tilde{\Theta}_{2}\right\|^{2}-\left\|\Theta_{2}^{*}\right\|^{2} .
$$

Substituting (58) into (57), we have

$$
\dot{V}_{2} \leq-z_{21}^{T} K_{2} z_{21}-s_{2}^{T} h_{2} s_{2}-\frac{\gamma_{2}}{2}\left\|\tilde{\Theta}_{2}\right\|^{2}+\frac{\gamma_{2}}{2}\left\|\Theta_{2}^{*}\right\|^{2} .
$$

Similar to the position loop, the approximation function is used to replace a symbolic function, the proof is the same as before (34). The inequality (59) can be written as

$$
\dot{V}_{2} \leq-\hbar_{2} V_{2}+\ell_{2}
$$

where $\hbar_{2}=\min \left\{\lambda_{\min }\left(K_{2}\right), h_{2}-(1 / 2), \gamma_{2} / 2\right\}$ and $\ell_{2}=\left(\gamma_{2} / 2\right)$ $\left\|\Theta_{2}^{*}\right\|^{2}+2 \eta_{2}^{2}$.

Consider the Lyapunov function candidate of the overall closed-loop system

$$
V=V_{1}+V_{2}
$$

Consider (35) and (60), differentiating $V$ yields

$$
\dot{V}=\dot{V}_{1}+\dot{V}_{2} \leq-\hbar V+\ell,
$$

where $\hbar=\min \left\{\lambda_{\min }\left(K_{1}+c_{1}\right), h_{1}-(1 / 2), \gamma_{1} / 2, \lambda_{\min }\left(K_{2}\right), h_{2}\right.$ $\left.-(1 / 2), \gamma_{2} / 2\right\}$ and $\ell=\left(\gamma_{1} / 2\right)\left\|\Theta_{1}^{*}\right\|^{2}+\left(\gamma_{2} / 2\right)\left\|\Theta_{2}^{*}\right\|^{2}+2 \eta_{1}^{2}+$ $2 \eta_{2}^{2}$

From (62), if appropriate parameter which satisfying $h_{1}>(1 / 2)$ and $h_{2}>(1 / 2)$ is chosen, all the closed-loop system signals are bounded under the action of developed controller (25) and (53) according to Lemma 1. Thus, all the tracking errors and the sliding mode surfaces are bounded. The UAH can track the desired trajectory without the effect of unknown disturbances and model uncertainties. Then, $z_{11}, s_{1}, \tilde{\Theta}_{1}, z_{21}, s_{2}$, and $\tilde{\Theta}_{2}$ are uniformly bounded according to Lemma 1. Then, proof is completed.

\section{Simulation Results}

In this section, the simulation results are presented to investigate the effectiveness of the proposed adaptive SMC scheme for the medium-scale UAH with model uncertainties and external unknown disturbances. The parameters of the medium-scale UAH are given as $m=800 \mathrm{~kg}, g=9.8 \mathrm{~m} / \mathrm{s}^{2}$, $\left(J_{x x}, J_{y y}, J_{z z}\right)=(360,780,600)\left(\mathrm{kg} \cdot \mathrm{m}^{2}\right)$. The parameter uncertainty of the simulation system is set to $20 \%$, and the disturbance is chosen as $d_{1}=[1.5 \sin (0.4 t), 1.2 \sin (0.4 t)$, 

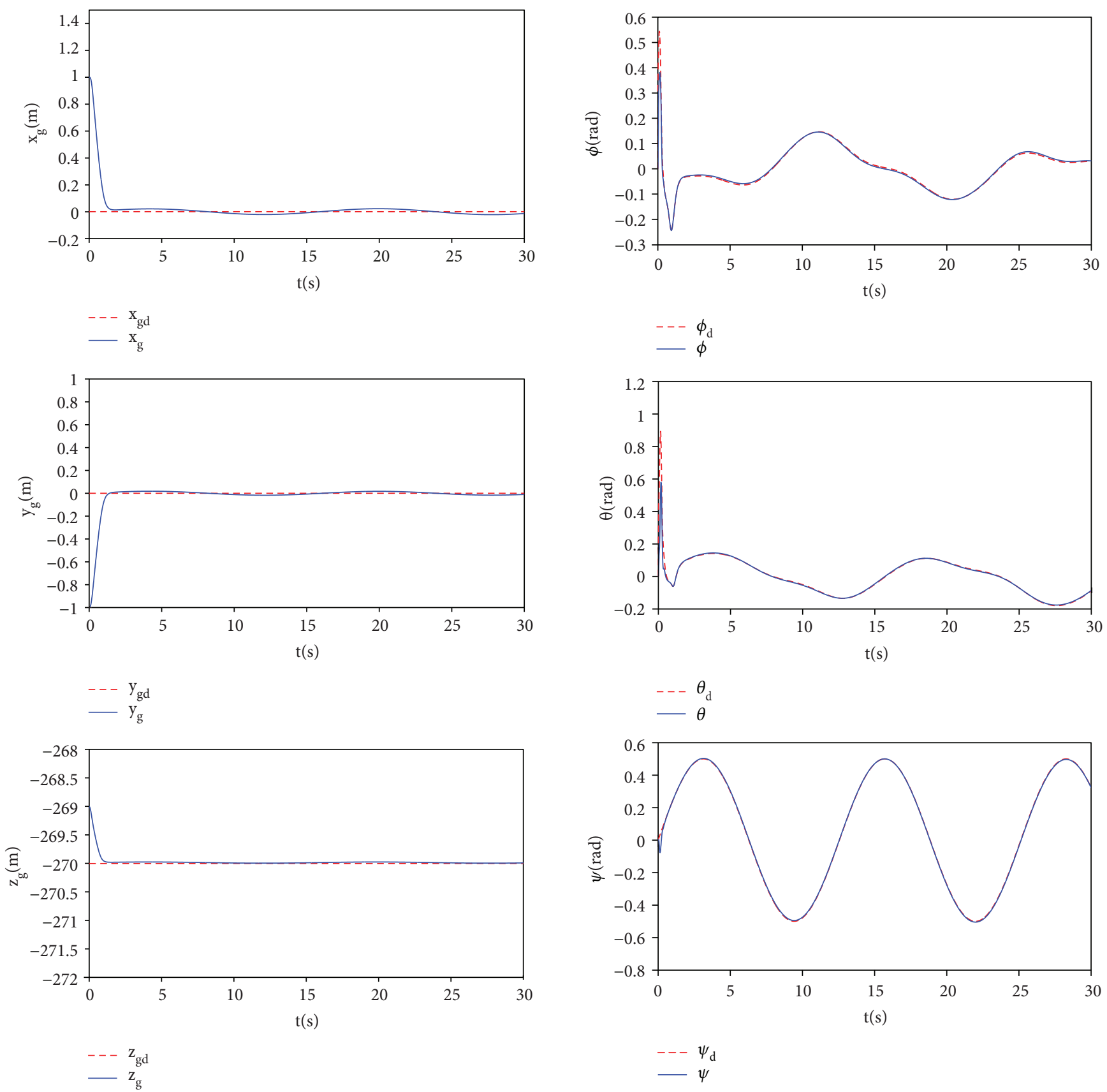

FIGURE 1: Tracking result of the position control for Case 1.

$1.2 \sin (0.4 t)]^{T}$ and $d_{2}=[2.5 \sin (0.3 t), 1.2 \sin (0.5 t), 1.8$ $\sin (0.2 t)]^{T}$.

In order to illustrate the effectiveness of the proposed control scheme, two different simulation cases are studied based on these state conditions.

Case 1. The initial state conditions are assumed as $x_{0}=1 \mathrm{~m}$, $y_{0}=-1 \mathrm{~m}$, and $z_{0}=-269 \mathrm{~m}$, and the desired trajectory is given by $x_{1 d}=\left[\begin{array}{lll}0 & 0 & -270\end{array}\right]^{T} \mathrm{~m}$ and $\psi_{d}=0.5 \sin (0.5 t)$ rad. The design parameters are chosen as $K_{1}=\operatorname{diag}\{5,5$, $10\}, K_{2}=\operatorname{diag}\{15,10,10\}, c_{1}=\operatorname{diag}\{5,5,2\}, c_{2}=\operatorname{diag}\{1$, $1,1\}, h_{1}=0.55, h_{2}=1, \eta_{1}=10$, and $\eta_{2}=50$, and the updated

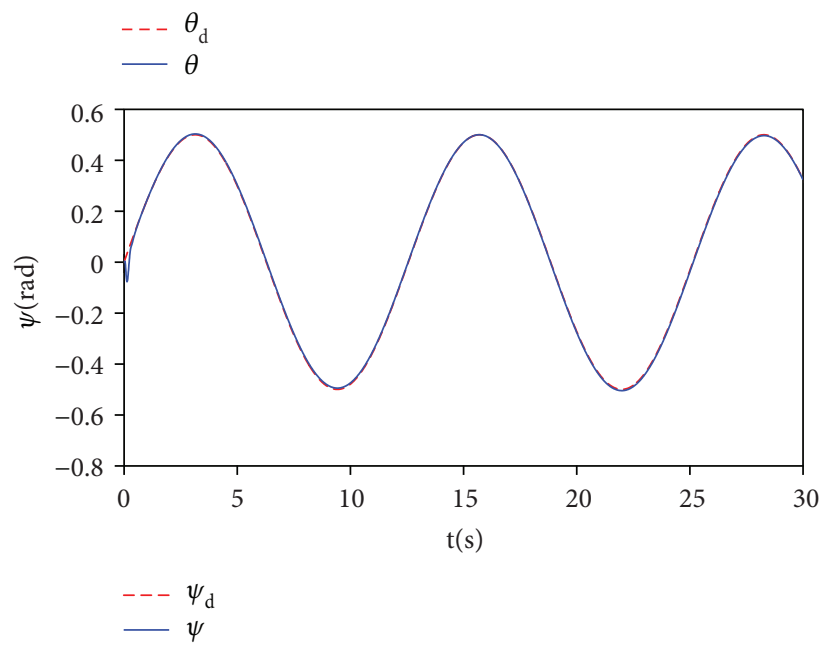

FIgURe 2: Tracking result of the attitude control for Case 1.

law of NN parameters is chosen as $\Gamma_{1}=5, \gamma_{1}=2, \Gamma_{2}=5$, and $\gamma_{2}=2$.

Figure 1 illustrates the tracking results of the position control, and Figure 2 illustrates tracking results of the attitude control for Case 1. The control inputs of thrust and moments for Case 1 are shown in Figure 3. From Figures 1 and 2, we can see that the tracking performance is good and tracking error quickly converges. From Figure 3, we note that the control inputs are bounded and convergent.

Case 2. The initial state conditions are assumed as $x_{0}=0.5 \mathrm{~m}$, $y_{0}=-0.5 \mathrm{~m}$, and $z_{0}=-265 \mathrm{~m}$, and the desired trajectory 

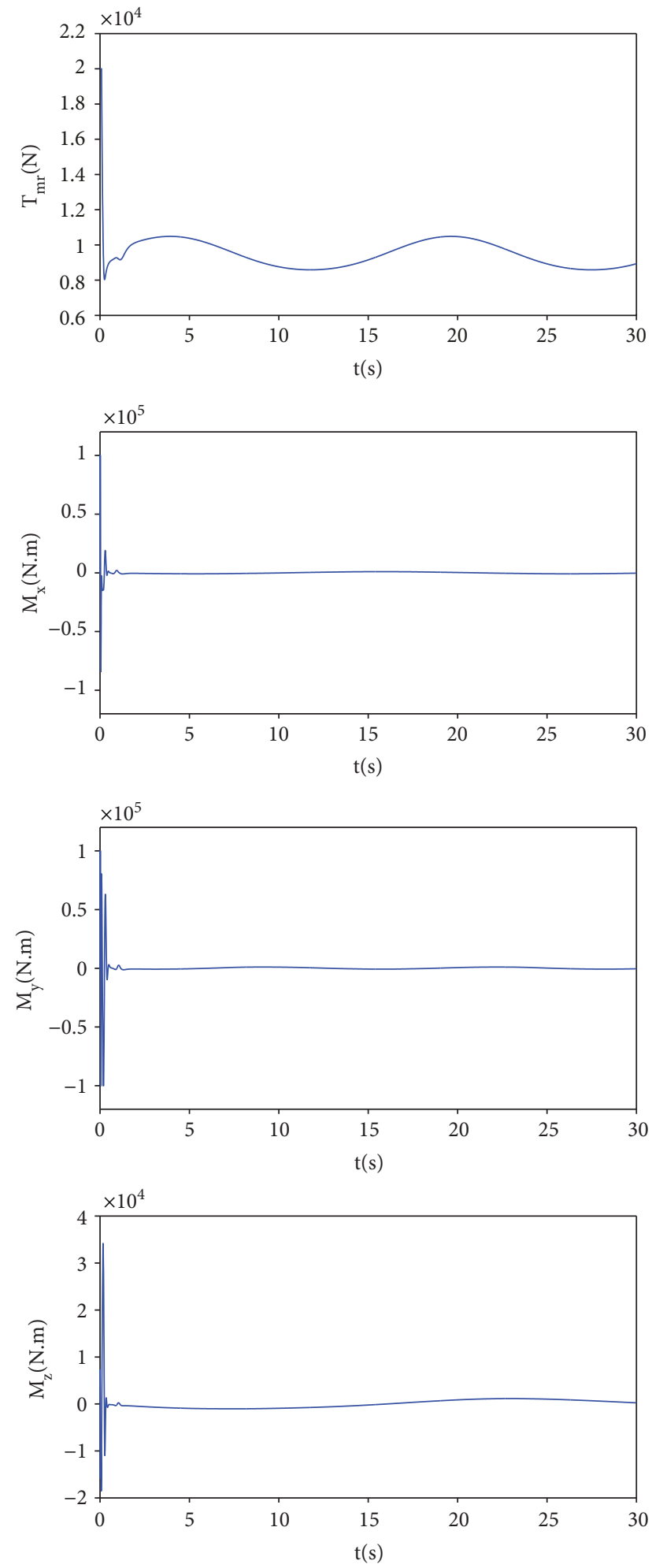

FIgURE 3: The control inputs of thrust and moments for Case 1.

is given by $x_{1 d}=\left[\begin{array}{lll}5 \sin (0.5 t) & 4 \sin (0.4 t) & -270\end{array}\right]^{T} \mathrm{~m}$ and $\psi_{d}=0.2 \mathrm{rad}$. The design parameters are chosen as $K_{1}=\operatorname{diag}$ $\{5,5,5\}, K_{2}=\operatorname{diag}\{20,5,30\}, c_{1}=\operatorname{diag}\{5,5,2\}, c_{2}=\operatorname{diag}$ $\{1,1,1\}, h_{1}=0.55, h_{2}=1, \eta_{1}=5$, and $\eta_{2}=50$, and the updated law of NN parameters is chosen as $\Gamma_{1}=5, \gamma_{1}=$
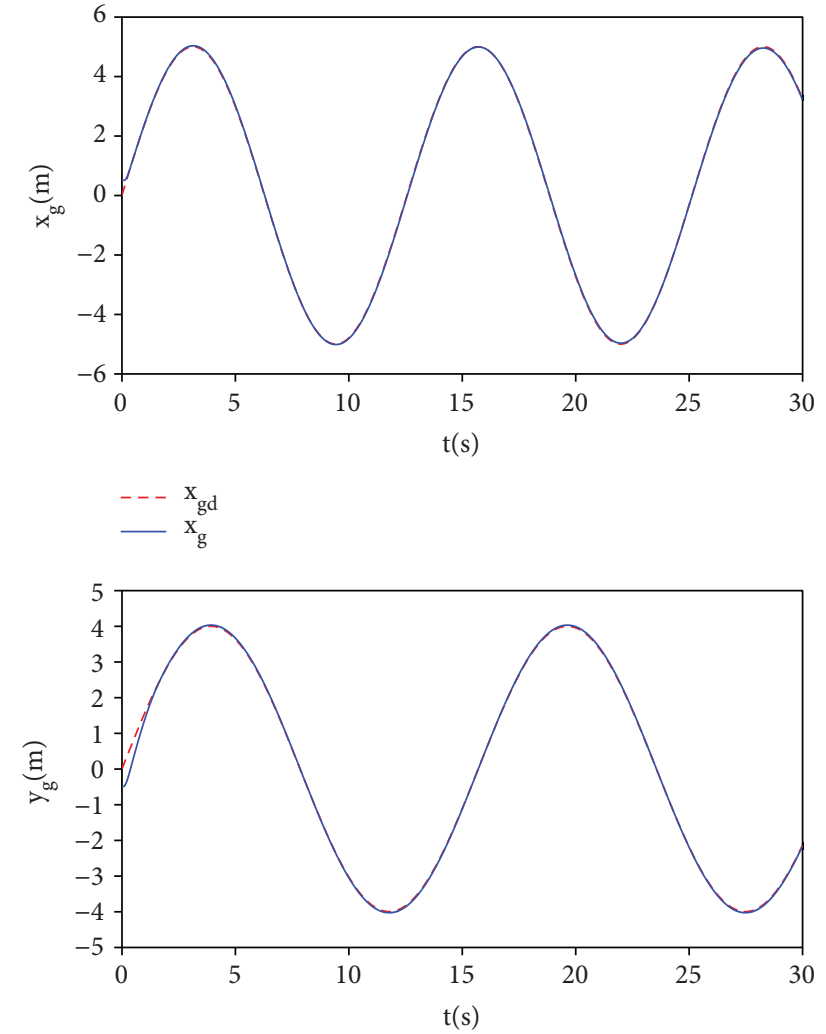

$--y_{g d}$
$-y_{g}$

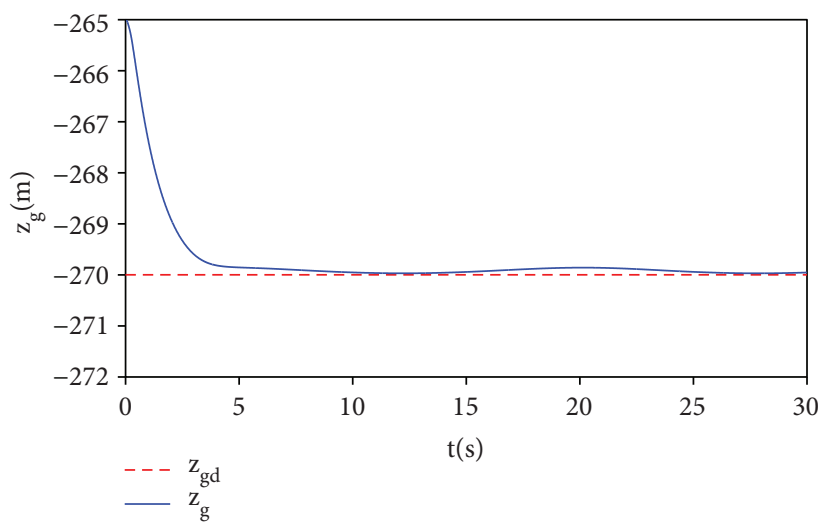

FIgURe 4: Tracking result of the position control for Case 2.

2, $\Gamma_{2}=5$, and $\gamma_{2}=2$. The tracking results of the position control and attitude control for Case 2 are shown in Figures 4 and 5, respectively. According to Figures 4 and 5, the simulation results show that the tracking errors are small and the output can rapidly track the reference signal. The control inputs of thrust and moments for Case 2 are presented in Figure 6. From Figure 6, the bounded control inputs are observed.

It can be observed from tracking results for two cases that the position and the attitude angles can track the desired trajectory in a satisfactory way in the presence of system uncertainties and external unknown disturbances. We note that 

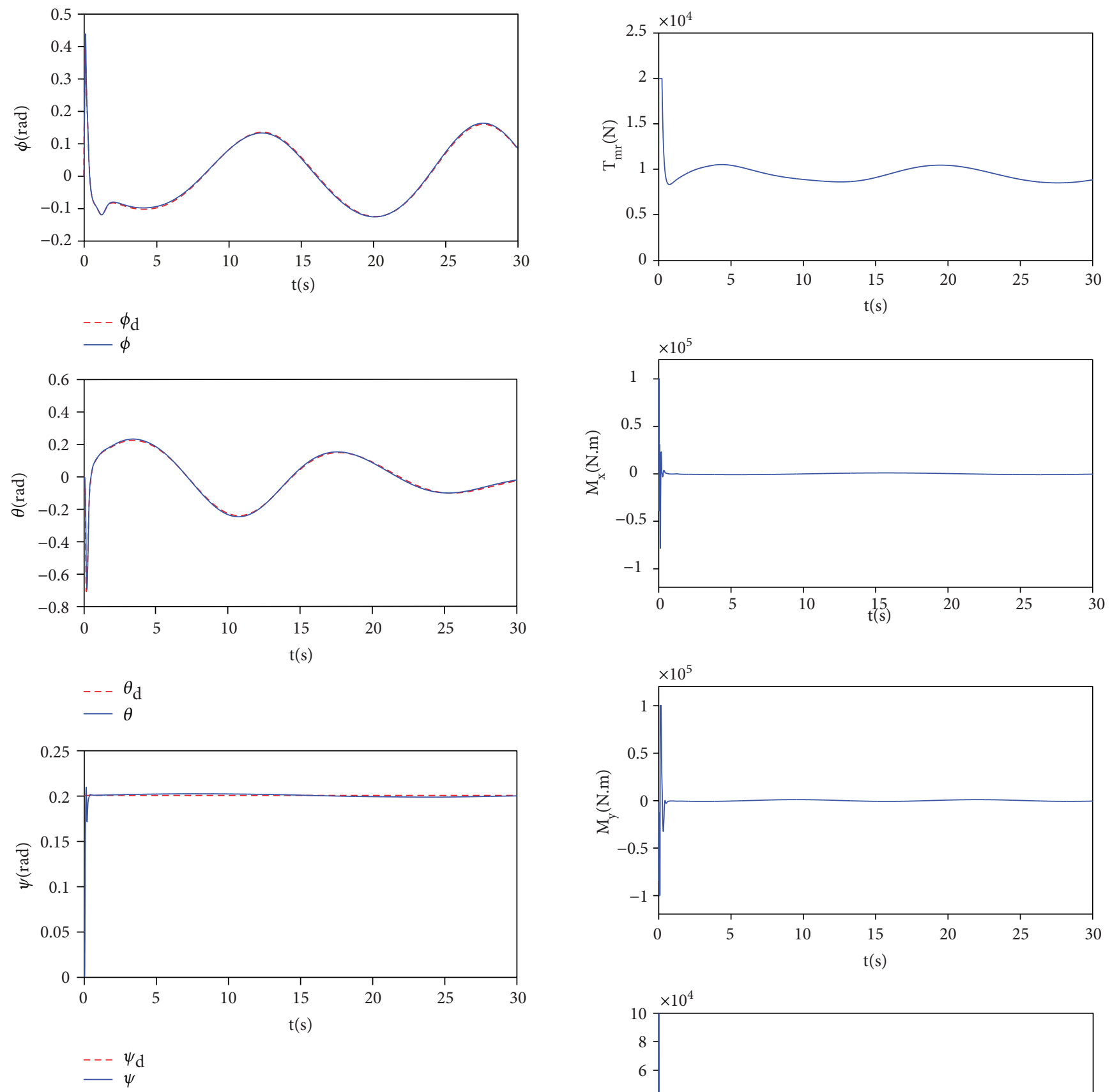

FIGURE 5: Tracking result of the attitude control for Case 2.

the system has strong robustness. From Figures 3 and 6, it is obvious that the control input commands are bounded and convergent. Thus, we can conclude that the proposed adaptive SMC tracking control strategy is effective for the UAH with system uncertainties and disturbances.

\section{Conclusion}

In this paper, an adaptive sliding controller has been developed for the UAH system on the basis of the neural network and backstepping, which can make the UAH tracks the desired trajectory accurately even if it has the parameter

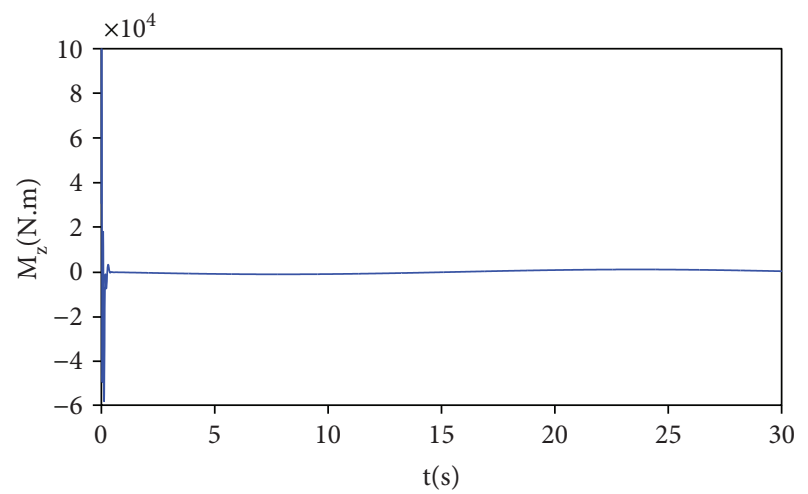

Figure 6: The control inputs of thrust and moments for Case 2.

uncertainties and the external disturbances. Firstly, a mathematical model has been established and simplified appropriately. Then, an adaptive sliding mode controller has been designed for tackling the unknown disturbances. In addition, 
the RBFNN has been utilized to estimate the UAH system uncertainty. Finally, the proposed control scheme can ensure that all signals of the closed-loop system are uniformly bounded by using the Lyapunov analysis method. The effectiveness of the method has been verified by simulation.

\section{Data Availability}

The data used to support the findings of this study are available from the corresponding author upon request.

\section{Conflicts of Interest}

The authors declare that they have no conflicts of interest.

\section{Acknowledgments}

This work was supported in part by the National Natural Science Foundation of China under Grant no. 61533008, in part by the Natural Science Foundation of Jiangsu Province of China (BK20171417), and in part by the Aeronautical Science Foundation of China under Grant no. 20165752049.

\section{References}

[1] Y. He, H. Pei, H. Zhou, and T. Sun, "Adaptive backsteppingbased robust tracking control of unmanned helicopters with disturbances," Control Theory \& Applications, vol. 30, no. 7, pp. 834-843, 2013.

[2] K. Yan, Q. Wu, and M. Chen, "Robust adaptive backstepping control for unmanned autonomous helicopter with flapping dynamics," in 2017 13th IEEE International Conference on Control \& Automation (ICCA), pp. 1027-1032, Ohrid, Macedonia, July 2017.

[3] S. Puntunan and M. Parnichkun, "Online self-tuning precompensation for a PID heading control of a flying robot," International Journal of Advanced Robotic Systems, vol. 3, no. 4, pp. 323-330, 2006.

[4] H. Liu, G. Lu, and Y. Zhong, "Robust LQR attitude control of a 3-DOF laboratory helicopter for aggressive maneuvers," IEEE Transactions on Industrial Electronics, vol. 60, no. 10, pp. 4627-4636, 2013.

[5] G. Cai, B. M. Chen, X. Dong, and T. H. Lee, "Design and implementation of a robust and nonlinear flight control system for an unmanned helicopter," Mechatronics, vol. 21, no. 5, pp. 803-820, 2011.

[6] F. Leonard, A. Martini, and G. Abba, "Robust nonlinear controls of model-scale helicopters under lateral and vertical wind gusts," IEEE Transactions on Control Systems Technology, vol. 20, no. 1, pp. 154-163, 2012.

[7] C. Liu, W.-H. Chen, and J. Andrews, "Tracking control of small-scale helicopters using explicit nonlinear MPC augmented with disturbance observers," Control Engineering Practice, vol. 20, no. 3, pp. 258-268, 2012.

[8] M. Chen, Q. X. Wu, C. S. Jiang, and B. Jiang, "Guaranteed transient performance based control with input saturation for near space vehicles," Science China Information Sciences, vol. 57, no. 5, pp. 1-12, 2014.

[9] Y. Gao, X. Sun, and W. Wang, "Decentralized backstepping adaptive output tracking of large-scale stochastic nonlinear systems," Science China Information Sciences, vol. 60, no. 12, 2017.

[10] E. Frazzoli, M. A. Dahleh, and E. Feron, "Trajectory tracking control design for autonomous helicopters using a backstepping algorithm," in Proceedings of the 2000 American Control Conference. ACC (IEEE Cat. No.00CH36334), vol. 6, pp. 4102-4107, Chicago, IL, USA, June 2000.

[11] B. Zhu and W. Huo, "Robust nonlinear control for a modelscaled helicopter with parameter uncertainties," Nonlinear Dynamics, vol. 73, no. 1-2, pp. 1139-1154, 2013.

[12] X.-Y. Lu and S. K. Spurgeon, "Robust sliding mode control of uncertain nonlinear systems," Systems \& Control Letters, vol. 32, no. 2, pp. 75-90, 1997.

[13] P. Li, X. Yu, X. Peng, Z. Zheng, and Y. Zhang, "Fault-tolerant cooperative control for multiple UAVs based on sliding mode techniques," Science China Information Sciences, vol. 60, no. 7, 2017.

[14] R. Xu and Ü. Özgüner, "Sliding mode control of a class of underactuated systems," Automatica, vol. 44, no. 1, pp. 233241, 2008.

[15] E. S. Espinoza, O. Garcia, I. Lugo, P. Ordaz, A. Malo, and R. Lozano, "Modeling and sliding mode control of a micro helicopter-airplane system," Journal of Intelligent and Robotic Systems, vol. 73, no. 1-4, pp. 469-486, 2014.

[16] D. Lee, H. Jin Kim, and S. Sastry, "Feedback linearization vs. adaptive sliding mode control for a quadrotor helicopter," International Journal of Control, Automation and Systems, vol. 7, no. 3, pp. 419-428, 2009.

[17] M. Chen, G. Tao, and B. Jiang, "Dynamic surface control using neural networks for a class of uncertain nonlinear systems with input saturation," IEEE Transactions on Neural Networks and Learning Systems, vol. 26, no. 9, pp. 2086-2097, 2015.

[18] J. Na, X. Ren, C. Shang, and Y. Guo, “Adaptive neural network predictive control for nonlinear pure feedback systems with input delay," Journal of Process Control, vol. 22, no. 1, pp. 194-206, 2012.

[19] J. Na, X. Ren, G. Herrmann, and Z. Qiao, “Adaptive neural dynamic surface control for servo systems with unknown dead-zone," Control Engineering Practice, vol. 19, no. 11, pp. 1328-1343, 2011.

[20] J. Na, X. Ren, and D. Zheng, “Adaptive control for nonlinear pure-feedback systems with high-order sliding mode observer," IEEE Transactions on Neural Networks and Learning Systems, vol. 24, no. 3, pp. 370-382, 2013.

[21] D. Nodland, H. Zargarzadeh, and S. Jagannathan, "Neural network-based optimal adaptive output feedback control of a helicopter UAV," IEEE Transactions on Neural Networks and Learning Systems, vol. 24, no. 7, pp. 1061-1073, 2013.

[22] T. Lee and Y. Kim, "Nonlinear adaptive flight control using backstepping and neural networks controller," Journal of Guidance, Control, and Dynamics, vol. 24, no. 4, pp. 675-682, 2001.

[23] J. Liu, A. Wu, R. Guo, and X. Fang, "Helicopter dynamic inversion flight control based on neural network," Flight Dynamics, vol. 32, no. 3, pp. 218-221, 2014.

[24] B. Xu, Z. Shi, C. Yang, and F. Sun, "Composite neural dynamic surface control of a class of uncertain nonlinear systems in strict-feedback form," IEEE Transactions on Cybernetics, vol. 44, no. 12, pp. 2626-2634, 2014.

[25] B. Xu, C. Yang, and Y. Pan, "Global neural dynamic surface tracking control of strict-feedback systems with application to hypersonic flight vehicle," IEEE Transactions on Neural 
Networks and Learning Systems, vol. 26, no. 10, pp. 2563-2575, 2015.

[26] G. Cai, B. Chen, and T. Lee, Unmanned Rotorcraft Systems, Springer, Heidelberg, 2011.

[27] Y. Yang, Helicopter Flight Control, National Defense Industry Press, 2007.

[28] X. Sun, Y. Fang, and N. Sun, "Backstepping-based adaptive attitude and height control of a small-scale unmanned helicopter," Control Theory and Applications, vol. 29, no. 3, pp. 381-388, 2012.

[29] M. Chen, P. Shi, and C.-C. Lim, "Adaptive neural faulttolerant control of a 3-DOF model helicopter system," IEEE Transactions on Systems, Man, and Cybernetics: Systems, vol. 46, no. 2, pp. 260-270, 2016.

[30] S. S. Ge and C. Wang, "Adaptive neural control of uncertain MIMO nonlinear systems," IEEE Transactions on Neural Networks, vol. 15, no. 3, pp. 674-692, 2004.

[31] S. S. Ge, B. Ren, and M. Chen, "Robust attitude control of helicopters with actuator dynamics using neural networks," IET Control Theory and Applications, vol. 4, no. 12, pp. 28372854, 2010.

[32] M. Chen and S. S. Ge, "Direct adaptive neural control for a class of uncertain nonaffine nonlinear systems based on disturbance observer," IEEE Transactions on Cybernetics, vol. 43, no. 4, pp. 1213-1225, 2013.

[33] R. Li, M. Chen, and Q. Wu, "Adaptive neural tracking control for uncertain nonlinear systems with input and output constraints using disturbance observer," Neurocomputing, vol. 235, pp. 27-37, 2017.

[34] R. Xu and U. Ozguner, "Sliding mode control of a quadrotor helicopter," in Proceedings of the 45th IEEE Conference on Decision and Control, pp. 4957-4962, San Diego, CA, USA, December 2006.

[35] X. Fang, A. Wu, and N. Dong, “Adaptive backstepping-based trajectory control of an unmanned helicopter in presence of wind gusts," Journal of Chinese Inertial Technology, vol. 23, no. 1, pp. 59-65, 2015. 


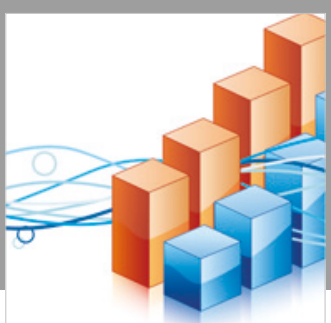

Advances in

Operations Research

\section{-n-m}
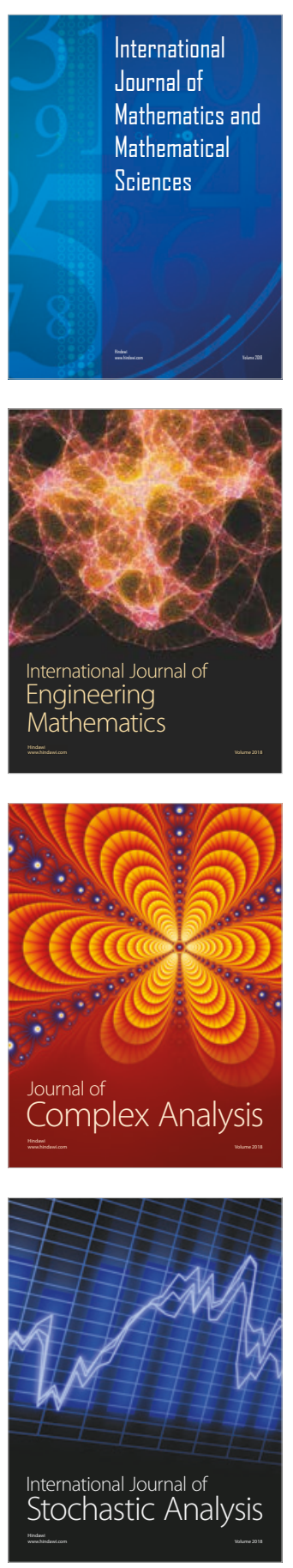
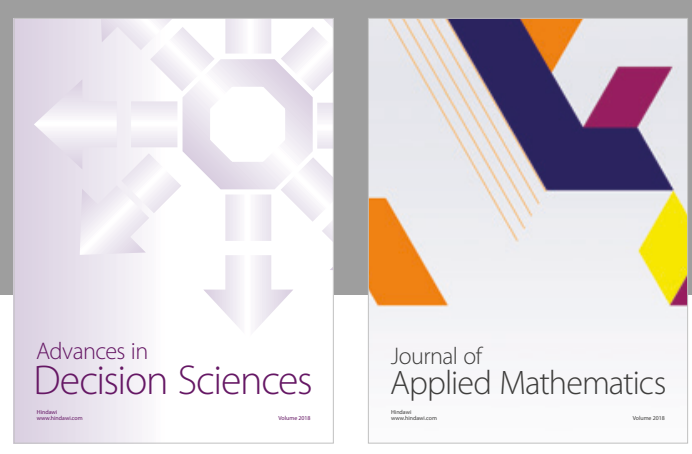

Journal of

Applied Mathematics
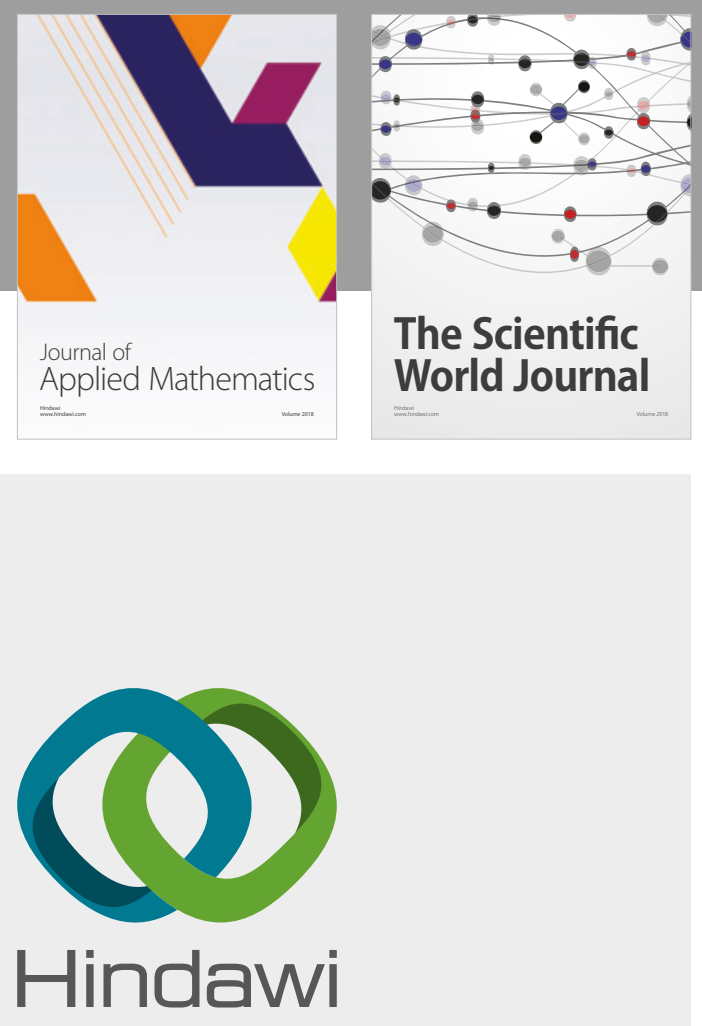

Submit your manuscripts at

www.hindawi.com

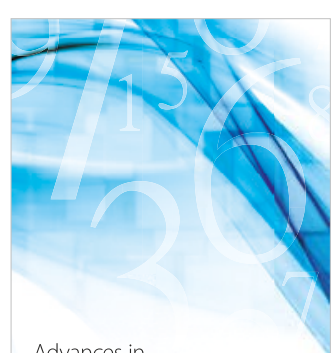

Advances in
Numerical Analysis
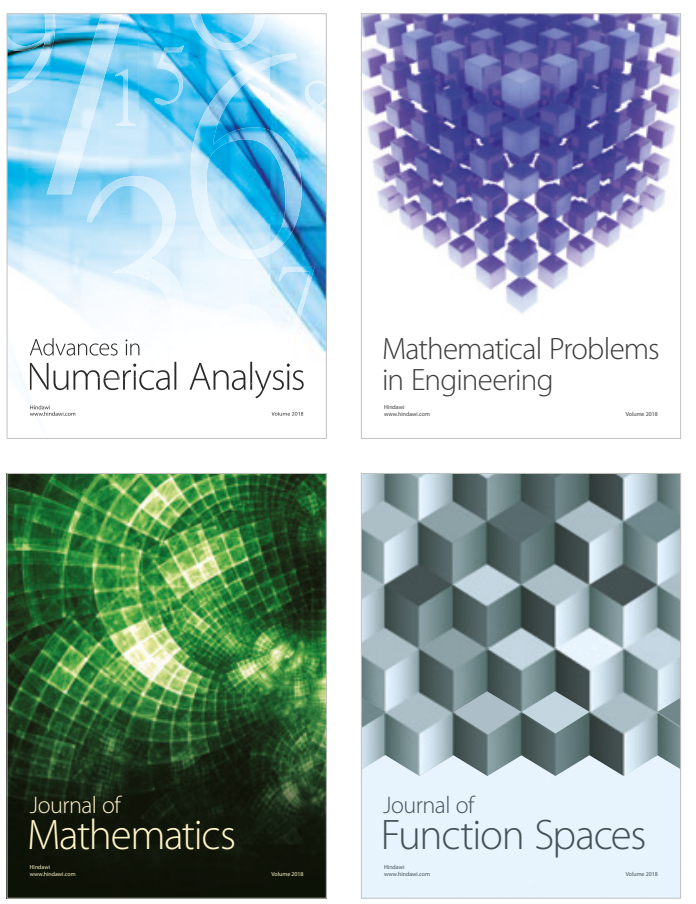

Mathematical Problems in Engineering

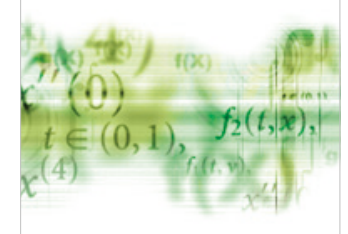

International Journal of

Differential Equations

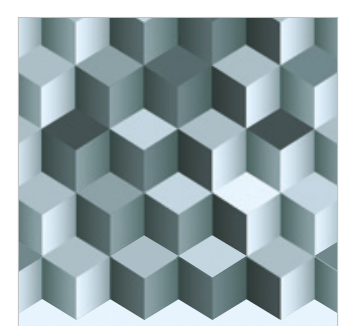

Journal of

Function Spaces
The Scientific

World Journal

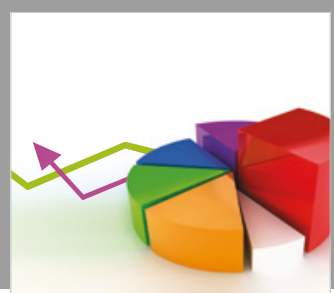

Journal of

Probability and Statistics
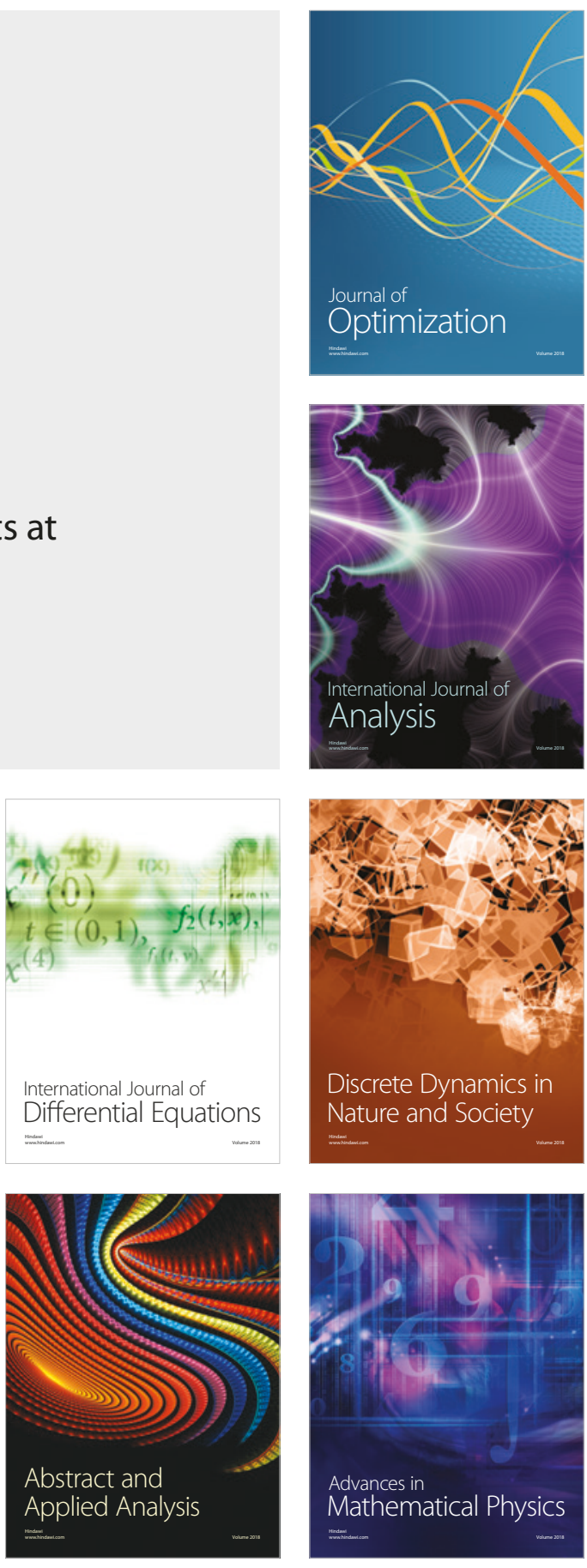